\title{
Spin-polarized electron tunneling between charge-density-wave metals
}

\author{
T. Ekino ${ }^{1}$, A.M. Gabovich², and A.I. Voitenko ${ }^{2}$ \\ ${ }^{1}$ Hiroshima University, Faculty of Integrated Arts and Sciences, 1-7-1 Kagamiyama, \\ Higashi-Hiroshima, 739-8521, Japan \\ ${ }^{2}$ Crystal Physics Department, Institute of Physics, National Academy of Sciences \\ 46 Prospekt Nauki, Kiev, 03028 Ukraine \\ E-mail: collphen@iop.kiev.ua
}

Received April 16, 2004

\begin{abstract}
For junctions between metals partially gapped by charge density waves (CDWs), the quasiparticle tunnel currents $J(V)$ and conductances $G(V)$ in external magnetic fields $H$ are calculated as functions of $H$, the bias voltage $V$, temperature $T$, the dielectric gaps $\Sigma$, and the gapped portions $\mu$ of the Fermi surface (FS). The paramagnetic effect of $H$ is taken into account, whereas orbital effects are neglected. General expressions are obtained for different CDW metal electrodes. Analytical formulas are obtained for $T=0$. Explicit numerical calculations are carried out for symmetrical junctions. The results are substantially unlike those for junctions between superconductors. It is shown that due to the interplay between quasiparticles from nested and non-nested FS sections the junction properties involve features appropriate to both symmetrical and asymmetrical setups. In particular, for $H=0$ discontinuities at $e V= \pm 2 \Sigma$ and square-root singularities at $e V= \pm \Sigma$ should coexist. Here $e$ is the elementary charge. For $H \neq 0$ the former remain intact, while the latter split. It is suggested to use the splitting as a verification of the CDW nature of the pseudogap in high- $T_{C}$ superconducting oxides.
\end{abstract}

PACS: 71.45.Lr, 73.40.Gk, 75.20.En, 75.47.Np

\section{Introduction}

Instabilities of the parent metallic electron spectrum leading to a formation of charge density waves (CDWs) [1-3] are in some sense similar to the superconducting Cooper pairing phenomenon [4]. Namely, although $\mathrm{CO}^{-}$ herent properties of the reconstructed low-temperature, low- $T$, phases are quite different, the resulting gapping of the Fermi surface (FS) due to many-body correlations is described by the same equations, at least in the weak-coupling limit. Therefore, the so-called semiconducting aspects of both superconductors and excitonic $[1,2]$ or Peierls [3] insulators are analogous. Nevertheless, as has been demonstrated previously [5], they are by no means identical. It is worthwhile noting that the quasiparticle electron density of states (DOS) of conventional nondegenerate semiconductors, adequately described by the one-body band theory, is nonsingular [6], contrary to what happens in the models both for superconductors [4] and many-body insulators [1-3].
In this publication we want to call attention once more to the quasiparticle tunneling between metals partially gapped by CDWs (CDWMs). The expressions for the tunnel current-voltage (I-V) characteristics $J(V)$ in the general case of different CDWM electrodes are obtained, and a number of practically important particular cases are considered in more detail. Their analysis shows that due to the coexistence of gapped and nongapped FS sections, the $\mathrm{I}-\mathrm{V}$ characteristics of tunnel junctions with CDWMs on both sides of the potential barrier possess some unconventional features. They are analogous to those observed in the setup where one of the electrodes is a normal metal without any electron spectrum distortion and the other one is a partially-gapped CDW conductor [5,7].

If an external magnetic field $H$ is applied, the dual nature of the partially-gapped CDWM should result in the Zeeman (spin) splitting of the peaks in the conductance-voltage $(\mathrm{G}-\mathrm{V})$ characteristics $G(V)$, 
which, e.g., in the case of superconductivity are appropriate to $\mathrm{S}-\mathrm{I}-\mathrm{N}$ junctions rather than to the $\mathrm{S}-\mathrm{I}-\mathrm{S}$ ones (compare with Refs. 4,8-10). In this article we obtain the corresponding expressions for $G(V)$ in a symmetrical CDWM-I-CDWM structure and demonstrate the existence of the peak splitting. On this basis, relevant inferences are drawn for recognized CDW materials and high- $T_{c}$ cuprates, highly suspected to belong to this class $[11,12]$.

\section{Theory}

As has long been understood (see review [13]), the $\mathrm{C}-\mathrm{V}$ characteristics for tunneling between two superconductors in an external magnetic field $H$, which induces the Zeeman splitting of the electronic DOS due to the Pauli paramagnetism of electrons, nevertheless, does not exhibit splitting of the gap-related peaks. The nonexistance of the splitting in $\mathrm{S}^{\prime}-\mathrm{I}-\mathrm{S}$ junctions is explained by equal shifts in energy of electron subbands possessing the same spin projection value on both sides of the barrier and the conservation of spin direction while tunneling in the absence of the spin-orbital effect [14]. On the other hand, tunneling across $\mathrm{S}-\mathrm{I}-\mathrm{N}$ junctions reveals such a splitting, because in this situation $G(V)$ is proportional to the superconducting and normal electron DOSs shifted with respect to each other in the magnetic field [4,8-10].

As concerns the paramagnetic properties, a CDWM described either by the excitonic [1,2] or Peierls [3] models is quite similar [15-18] to an $s$-wave Bardeen-Cooper-Schrieffer (BCS) superconductor $[13,14]$. This means that for $H$ not exceeding a certain value, mathematically analogous to the Clogston-Chandrasekhar paramagnetic limit [4], the CDW gap $\Sigma(T)$ may be considered as a BCS-like one, not dependent on $H$. Such an expectation is supported by experiment. For example, the destructive influence of $H$ on the critical temperature, $T_{d}$, of the structural phase transition was observed to be extremely small at low fields for such different substances with CDWs as the A15 compound $\mathrm{V}_{3} \mathrm{Si}$ [19] $\left(T_{d}=20.15 \mathrm{~K}\right.$ at $H=0$ and is reduced by $-0.6 \mathrm{~K}$ at $H=156 \mathrm{kOe})$ and the quasi-one-dimensional organic metal $\mathrm{Per}_{2}\left[\mathrm{Au}(\mathrm{mnt})_{2}\right]$ [20], and could not be detected for any other CDW compounds.

On the other hand, in the following analysis we are going to completely ignore the diamagnetic response of the CDWM. The experimental reason for this neglect was cited above. From the theoretical point of view, it may be justified as follows. Due to the different type of long-range order in comparison to that for superconductors, the Meissner effect is absent in excitonic or Peierls insulators $[1,2,11,12]$, although other interesting coherent phenomena may occur
[21-24]. The more conventional orbital effects of the magnetic field should exist, but their influence on the CDW phase is not destructive. On the contrary, according to Refs. 25, 26, the inevitable paramagnetic effects are augmented by diamagnetic ones, favorable to CDWs due to the reduction of the electron spectrum dimensionality for large $H$ [4]. These considerations are supported by recent experiments for the organic metal $\alpha$-(BEDT-TTF $)_{2} \mathrm{KHg}(\mathrm{SCN})_{4}$ [27], where a series of phase transitions between subphases with different values of the nesting vector $\mathbf{Q}$ was observed. A stabilization of CDWs by the restricted orbital motion in the magnetic field is analogous to the emergence of field-induced spin-density waves (SDWs) in (TMTSF) ${ }_{2} \mathrm{X}$ organic salts [28]. Therefore, this phenomenon, which preserves CDWs, would be helpful for the spin splitting of CDW-driven peaks in $G(V)$, although it might make the interpretation of the spectra more ambiguous.

\section{1. $C D W$ metal}

\subsubsection{Zero magnetic field}

The starting point of our approach is the mean-field Hamiltonian of the partially-gapped superconducting CDW metal proposed by Bilbro and McMillan [29]. For our current purposes we need a simpler case of a normal CDW metal, which can be obtained from the original model when the superconducting gap is identically zero [5]. According to this model, the FS of the CDWM is split into degenerate (nested, $i=1,2$ ) and nondegenerate (non-nested, $i=3$ ) sections. For the former, the bare quasiparticle spectrum branches reckoned from a common Fermi level are linked by the relation

$$
\xi_{1}(\mathbf{p})=-\xi_{2}(\mathbf{p}+\mathbf{Q}),
$$

where $\mathbf{Q}$ is the $\mathrm{CDW}$ vector. Due to the interaction of quasiparticles from different $(i=1,2)$ nested FS sections, a many-body correlation (leading to a pairing, which is a close analog of Cooper pairing) appears between them. The CDW pairing can be described by a dielectric order parameter $\tilde{\Sigma}$, and a relevant dielectric gap $\Sigma$ emerges at both nested sections. If this interaction is mainly of a Coulomb origin [1,2], and the branches $\xi_{1,2}(\mathbf{p})$ represent the electron and hole bands, respectively, the CDW gapping corresponds to the excitonic insulator. Another possibility appears if the degenerate spectrum $\xi_{1,2}(\mathbf{p})$ is quasi-one-dimensional and the quasiparticle interaction is mediated by phonons. Then CDW gapping results in an emergence of the Peierls insulator state [3]. In both CDW cases, the coupling occurs between quasiparticles with oppositely directed spins (singlet pairing). Those alternatives can be considered in the framework of the same approach. The rest of the FS remains undis- 
torted by CDWs and is described by the nondegenerate electron spectrum branch $\xi_{3}(\mathbf{p})$. The portion of the FS gapped by the CDW instability (partial gapping) is determined by the dielectric-gapping (dielectrization) parameter

$$
\mu=N_{d 0}(0) / N_{0}(0),
$$

where $N_{0}(0)=N_{n 0}(0)+N_{d 0}(0)$ is the total initial (above $T_{d}$ ) electronic DOS on the FS, and $N_{d 0}(0)$ and $N_{n 0}(0)$ are the relevant DOSs on the degenerate $(d)$ and nondegenerate $(n)$ FS sections, respectively.

In principle, CDWs may be commensurate or incommensurate with the background crystal lattice. In the excitonic insulator model, the Coulomb-induced distortion below the transition temperature, $T_{d}$, is commensurate. Moreover, the phase of the order parameter in excitonic insulators is always pinned $[30,31]$ and $\tilde{\Sigma}$ is an either positive or negative quantity $[2,11,12,32,33]$. On the other hand, in Peierls insulators, incommensurate CDWs with the order parameters $\tilde{\Sigma}=\Sigma \mathrm{e}^{i \varphi}$ may exhibit a rich dynamics, although in the direct current measurements they are usually pinned with arbitrarily frozen phases $\varphi[3,23]$.

In the framework of the approach adopted, the partially-gapped nonsuperconducting $\mathrm{CDW}$ metal $(C D W M)$ in an absence of the external magnetic field $H$ is described by the following temporal Green's functions $G_{i j}(\omega)$, where $i, j=1,2,3$ are the subscripts labeling the FS sections (see above):

$$
\begin{gathered}
G_{11}=G_{22} \equiv G_{d}, \\
G_{12}=G_{21} \equiv G_{c}, \\
G_{33} \equiv G_{n} .
\end{gathered}
$$

For all the other $i j$ combinations, $G_{i j}=0$. The function $G_{c}$ describes the electron-hole pairing. It is «normal» in a conventional sense [10], since it is not a product of either creation or annihilation operators only, but is, nevertheless, «anomalous» in analogy with the Gor'kov Green's function, because it is proportional to the CDW order parameter $\tilde{\Sigma}$. All technical details of the calculations and explicit expressions for the functions $G_{d}, G_{c}$, and $G_{n}$ can be found in our previous publications $[5,11,12]$.

Thus, one sees that in the Bilbro-McMillan model [29] adopted by us here and in accord with the division of the FS into $d$ and $n$ sections, the electron states are of two different kinds, dubbed from here on as $n$ and $d$ states. Nevertheless, it is important to comprehend that whatever the distinctions of the electron spectrum between quasiparticle branches, the whole system has a common chemical potential pinned to that of the metallic $n$ component and disposed inside the dielectric gap $\Sigma$ inherent to $d$ states. On the other hand, in the model of the doped excitonic insulator, the Fermi level is supposed to be located above or below the gap edge in its nearest neighborhood. Decades ago, a significant enhancement of the superconducting $T_{c}$ due to the DOS increase in the indicated energy range was expected to happen if such a situation would have been realized [2,34]. Unfortunately, these hopes turned out to be vain and in all compounds in which superconductivity and CDW gapping have been proved to coexist, the latter is detrimental to the former $[11,12]$.

So, in the reconstructed CDW phase below $T_{d}$, the density of the $n$ states, $N_{n}(\omega)$, may be considered as that in the absence of CDW gapping. Hereafter, the energy distance from the Fermi level will be denoted as $\omega$. Since the phenomena investigated in this publication are determined only by the states in a narrow shell near the FS, the energy dependence of $N_{n}(\omega)$ can be neglected, i.e. $N_{n}(\omega)=N_{n 0}(0)$. At the same time, the energy spectrum of the $d$ states involves a dielectric gap below $T_{d}$, so that its DOS takes on a superconducting-like appearance [2]

$$
N_{d}(\omega)=N_{d 0}(0) \frac{|\omega| \theta(|\omega|-\Sigma)}{\sqrt{\omega^{2}-\Sigma^{2}}} .
$$

As was shown by Frenkel [35,36] (see also extensive accounts in Refs. 9, 37), the tunnel current $J$ across the biased barrier between metal electrodes is given by an algebraic sum of the forward and backward components. The voltage dependence of $J$ is exponential for large and Ohmic for small $V$ [37]. We shall not extend the subsequent analysis beyond the Ohmic regime, since experimentally relevant dielectric gap energies fall within the range of $0.5 \mathrm{meV}<\Sigma<30 \mathrm{meV}$, whereas the deviations from Ohm's law, indicating a changeover to the Fowler-Nordheim tunneling, emerge when the electron energy gain $e V$ becomes comparable to the conduction band width $W$ for either of electrodes. (Hereafter, $e>0$ is the elementary charge). Really, in the majority of metals the energy $W$ exceeds $1 \mathrm{eV}$, so that the existing power-law corrections to conductances $G(V) \equiv d J / d V$ of the tunnel junctions involving such metals [9] are not important for our purposes. One can imagine, however, a hypothetical situation when more than one conduction band for each metal take part in tunneling, which is plausible for narrow-band metals. Then, additional features in $G(V)$ may appear [38].

In studying tunnel currents between CDW metals, we shall use a fruitful analogy between the latter and BCS superconductors. The standard way of handling tunneling between superconductors is the tunnel Hamiltonian method $[4,39,40]$. Then, $J(V)$ constitutes an integral of electronic DOSs and the difference be- 
tween the Fermi distribution functions for both electrodes [4,9,41]. Insofar as the conductivity in the superconducting state is Ohmic, one can introduce a unique parameter $R$ representing the junction resistance in the normal state. The quantity $R$ is inversely proportional to the averaged square of the tunnel matrix elements $[4,42]$. The same theoretical approach has been demonstrated to be applicable for tunneling between normal CDW metals and superconductors $[5,11,12]$.

\subsubsection{Nonzero magnetic field}

If the external magnetic field $H$ (the spatial $z$ axis is chosen to be aligned with $H$ ) is switched on, the $d$ and $n$ states exhibit quite different paramagnetic properties. To describe them properly, it is convenient at first to restrict the respective reasoning to the limiting situation $T=0$. For nonzero $H$ (hereafter we consider values of $H$ less than the paramagnetic limit $H_{p}$ for CDWM, see below), electrons with the spin projection $s_{z}=+1 / 2$ onto $H$ increase their energies by $\mu_{B}^{*} H$, while the electrons with the opposite spin direction, $s_{z}=-1 / 2$, reduce their energies by the same amount [43]. Here $\mu_{B}^{*}=e \hbar / 2 m^{*} c$ is the effective Bohr magneton, $\hbar$ is Planck's constant, $c$ is the velocity of light, and $m^{*}$ is the effective mass of the current carriers. Henceforth, quasiparticles with either spin direction will be labeled by «t» or «-». The quasiparticle level scheme is shown in Fig. 1.

Quasiparticles belonging to the $n$ section, for which the Fermi level segregates occupied and empty states, behave in a conventional manner inherent to normal metals $[43,44]$. Namely, the states from the «t» spin subband, for $H=0$ coinciding in energy with its «-» counterpart and, therefore, equally populated, shift upwards in energy. As a consequence of the quasiparticle transfer from the «+» to the «-» subband, the former becomes more and more depleted as $H$ increases, whereas the number of occupied states in the «-» subband rises simultaneously by the same amount. This field-induced spin-polarization results in a change of the chemical potential $\tilde{\mu}$, the latter coinciding with the Fermi energy $E_{F}$ of $n$ electrons at $T=0$. For small $H$, the relative corrections to $\tilde{\mu}$ are of the order of $\left(\mu_{B}^{*} H / E_{F}\right)^{2}$. Since we are interested in the effects when $\mu_{B}^{*} H$ is, at least, smaller than $\Sigma$, the inequality $\left(\mu_{B}^{*} H / E_{F}\right)^{2}<<1$ is valid and we may neglect the changes to $\tilde{\mu}$ altogether. It is necessary to remind that in itinerant Stoner ferromagnets this is not the case and $\tilde{\mu}$ is altered conspicuously by the respective spin polarizations [44] (see also an account of concomitant phenomena in Refs. 45, 46).

Thus, the electronic DOS and the Fermi distribution function in the phenomenological expressions for the tunnel current are not affected, in a first approxi-

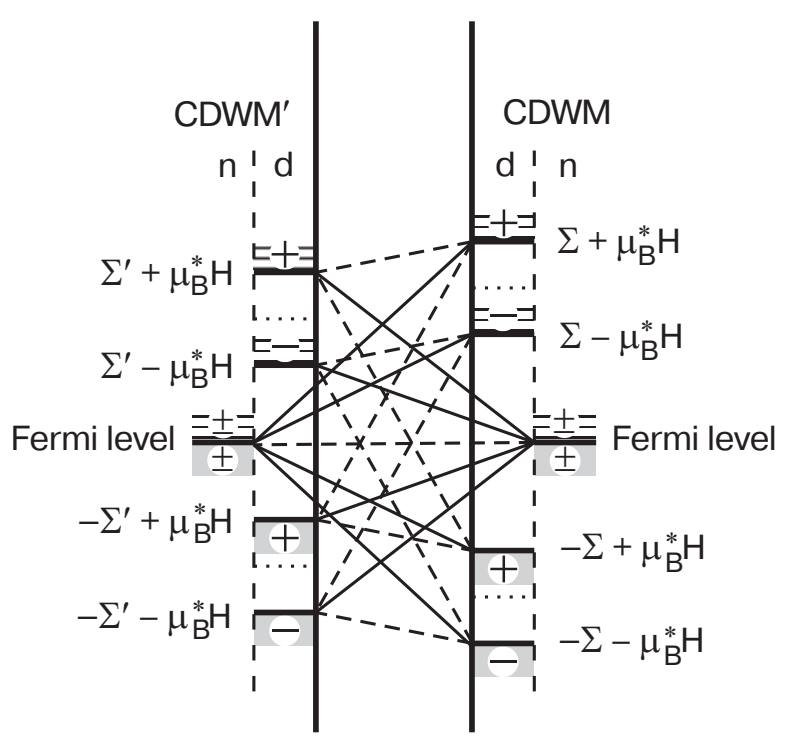

Fig. 1. The energy level scheme in a tunnel junction between partially gapped metals with charge-density waves (CDWs) affected by an external magnetic field $H$ at zero bias voltage. Quasiparticle energies for non-nested $(n)$ and nested ( $d$ ) Fermi surface (FS) sections are depicted separately on both sides of the insulating barrier. $\Sigma^{\prime}$ and $\Sigma$ are the CDW gaps on the left and on the right, respectively. $\mu_{B}^{*}$ is the Bohr magneton. «+» and «-» denote spin subbands with projections along $H$ and in the opposite direction. Dashed and solid lines correspond to the possible tunnel transitions without spin flipping, which make contribution to the current components that do not depend or depend on $H$, respectively.

mation, by magnetic fields, which are of the order of the energy parameters reflecting many-body gapping of the parent electron spectrum [4].

The paramagnetic splitting of quasiparticle states from the gapped FS sections can be examined analogously to that for superconductors $[13,14]$. The reason of the similarity is due to the fact that both CDW (electron-hole) and Cooper pairs are spin-singlet and therefore are prone to the destructive action of the Zeeman splitting $[15-18,47,48]$. As a result, the quasiparticles of the gapped «t» and «-» subbands shift in opposite directions in energy for $H \neq 0$. All spin-flip processes leading to the smearing of the ideal splitting are ignored hereafter, because we are interested in a qualitative picture only.

Once formed from the praphase, the electron system of the partially-gapped CDWM is stable against the influence of magnetic fields in the range defined below, the chemical potential $\tilde{\mu}$ being pinned at the original Fermi level.

When $T$ is finite, the Fermi distribution factors are no longer step-like functions and the thermally excited electron-like and hole-like quasiparticles appear above and below the gap $\Sigma$, respectively. At the same 
time, the chemical potential $\tilde{\mu}$ decreases with $T$, the relative correction being of the order $\left(k_{B} T / E_{F}\right)^{2}$ [4]. Here $k_{B}$ is the Boltzmann constant.

In what follows, we shall describe tunneling in a magnetic field $H \neq 0$ with the help of the Green's functions $G_{d}, G_{c}$, and $G_{n}$ mentioned above. The only modification, in comparison to the expressions of Refs. 5, 11, 12, is that now the number of Green's functions is doubled: six relevant $H$-dependent functions $G_{d s}, G_{c s}$, and $G_{n s}$ are denoted by an extra subscript $s= \pm$. They are functions of the relevant variables $\omega \mp \mu_{B}^{*} H$, the signs beeng inverse to those of $s$.

\subsubsection{Paramagnetic limit for CDWMs}

As has been indicated above, there exists Pauli limitation to CDW pairing similar to the Clogston-Chandrasekhar limit $[47,48]$ for superconductors. Since the Meissner orbital effect is absent in excitonic or Peierls insulators $[1,2,12]$, the paramagnetic effect manifests itself here just as it is. The evaluation of the paramagnetic limit $H_{p}$ for CDWMs is methodologically the same as in the case of BCS superconductors [4]. Specifically, one should compare the free energy of the partially-gapped phase $\delta F_{C D W M}$ with that of the paramagnetic state in the presence of the magnetic field $\delta F_{p}$. Moreover, we should take into account that the paramagnetic CDWM phase (the analog of the Sarma state in superconductors) is energetically unfavorable [49].

Since the gap $\Sigma(T)$ appears only on the $d$ (nested) FS sections, we obtain

$$
\left(\mu_{B}^{*} H_{p}\right)^{2}=\frac{\mu}{2} \Sigma_{0}^{2},
$$

where $\Sigma_{0}=\Sigma(T=0)$, so that

$$
H_{p}=\frac{\Sigma_{0}}{\mu_{B}^{*}} \sqrt{\frac{\mu}{2}} .
$$

The reduction of the actual $H_{p}$ in comparison to the limiting value of the complete gapping $(\mu=1)$ should be allowed for when comparatively analyzing orbital and spin effects in $\mathrm{CDW}$ substances. In particular, one should mention organic substances $\alpha-(\mathrm{ET})_{2} \mathrm{MHg}(\mathrm{SCN})_{4}$ $(\mathrm{M}=\mathrm{K}, \mathrm{Tl}, \mathrm{Rb}$, etc. $)[18,50-52]$.

\subsection{Current-voltage characterictics}

General expressions for quasiparticle currents across tunnel junctions between dissimilar CDWMs (a CDWM'-I-CDWM junction) are given in Appendix A. But the main features of the investigated phenomena are appropriate also to a simpler case of symmetrical junctions with identical CDWM electrodes. In the Bilbro-McMillan model [29] this means equality of the parameters $\tilde{\Sigma}$ and $\mu$. At the same time, the $\mathrm{I}-\mathrm{V}$ and $\mathrm{G}-\mathrm{V}$ characteristics become much less cumbersome. Indeed, the singularity positions, depending on the $\mathrm{CDWM}^{\prime}$ and CDWM gap magnitudes, merge in the symmetrical case, and certain pre-integral factors become equal.

But in making use of the emerging simplifying symmetry one should be very careful. For example, consider the pair $J_{c n+}$ and $J_{n c-}$. It is easy to ascertain from generic equations (A.3) or by analyzing the translation-containing symmetry properties (A.17) and (A.18) of the current components, that $J_{\mathrm{Cn+}}(\mathrm{eV})+$ $+J_{n c-}(e V)=0$. If we calculate the overall charge transfer regardless of the spin projection, the pairs of components like $J_{c n+}$ and $J_{n c-}$ may be neglected from the outset. However, it is a spin-splitting analysis. A premature mutual cancellation of the terms $J_{C n+}$ and $J_{n c-}$ would result in misleading results for each of the «+» and «-» components. Therefore, the best way to automatically avoid such traps is to add up the components with a certain $s$ separately before making the final comparison between $J_{+}(V)$ and $J_{-}(V)$.

Nevertheless, for symmetrical CDWM-I-CDWM junctions, we can exclude the $c d \pm$ and $d c \pm$ components from consideration, since $J_{c d s}(e V)=-J_{d c s}(e V)$ for each $s$.

Thus, a complete set of components of the quasiparticle tunnel current through a symmetrical CDWM-I-CDWM junction is as follows:

$J_{d d \pm}=\frac{\mu^{2}}{4 e R} \int_{-\infty}^{\infty} d \omega K\left|\omega_{\mp}\right| f\left(\omega_{\mp}, \Sigma\right)\left|\omega_{\mp}-e V\right| f\left(\omega_{\bar{\mp}}-e V, \Sigma\right)$

$$
\begin{gathered}
J_{c c \pm}=\frac{(\mu \Sigma)^{2}}{4 e R} \int_{-\infty}^{\infty} d \omega K \operatorname{sgn}\left(\omega_{\mp}\right) f\left(\omega_{\mp}, \Sigma\right) \operatorname{sgn}\left(\omega_{\mp}-e V\right) \times \\
\times f\left(\omega_{\mp}-e V, \Sigma\right)
\end{gathered}
$$

$$
J_{n n \pm}=\frac{(1-\mu)^{2} V}{2 R},
$$

$$
J_{d n \pm}=\frac{\mu(1-\mu)}{4 e R} \int_{-\infty}^{\infty} d \omega K\left|\omega_{\mp}\right| f\left(\omega_{\mp}, \Sigma\right)
$$

$$
J_{n d \pm}=\frac{\mu(1-\mu)}{4 e R} \int_{-\infty}^{\infty} d \omega K\left|\omega_{\mp}-e V\right| f\left(\omega_{\mp}-e V, \Sigma\right),
$$

$$
\begin{gathered}
J_{c n \pm}=\frac{\mu(1-\mu) \Sigma}{4 e R} \int_{-\infty}^{\infty} d \omega K \operatorname{sgn}\left(\omega_{\mp}\right) f\left(\omega_{\mp}, \Sigma\right), \quad \mathrm{S}(14) \\
J_{n c \pm}=\frac{\mu(1-\mu) \Sigma}{4 e R} \int_{-\infty}^{\infty} d \omega K \operatorname{sgn}\left(\omega_{\mp}-e V\right) f\left(\omega_{\mp}-e V, \Sigma\right),
\end{gathered}
$$


where the factor $K=K(\omega, e V)$, generated by the Fermi distributions of current carriers in both electrodes, and the tunnel resistance $R$ are determined by Eqs. (A.5) and (A.4), respectively.

For $T=0$, all the current components, except the trivial Ohmic term $J_{n n \pm}$, can be expressed in terms of elliptic integrals. The relevant expressions are given in Appendix B. For $T \neq 0$, numerical calculations were made (see the next Section).

\section{Calculations}

The representative quantities of the setup under investigation (a symmetrical CDWM-I-CDWM junction in a magnetic field) are as follows: the critical temperature of the $\mathrm{CDW}$ phase transition $T_{d}$ or, equivalently, the zero-temperature dielectric gap $\Sigma_{0}=(\pi / \gamma) T_{d}$, and the gapping parameter $\mu$ [Eq. (2)] in either electrode, the junction resistance $R$ [Eq. (A.4)], the temperature $T$, and the external magnetic field $H$. Here $\gamma=1.78 \ldots$ is the Euler constant. Hereafter, we use the dimensionless parameters: $t=k_{B} T / \Sigma_{0}$ and $h=\mu_{B}^{*} H / \Sigma_{0}$.

\subsection{Conductance-voltage characteristics}

It is well-known that the differential tunnel $\mathrm{G}-\mathrm{V}$ characteristics $d J(V) / d V$ are much more informative than the original $\mathrm{I}-\mathrm{V}$ characteristics $J(V)$, with the former acting as an amplifier of the gap-driven peculiarities [9]. In particular, the $\mathrm{G}-\mathrm{V}$ characteristics give direct information about an energy dependence of the electronic DOS, renormalized due to Cooper $[4,8,9,53]$ or $\mathrm{CDW}[5,54]$ pairings for junctions between «normal» electrodes and those having a «gapped» electron spectrum. Thus, for brevity, we shall confine ourselves below to the analysis of tunnel $\mathrm{G}-\mathrm{V}$ characteristics and introduce dimensionless spin-dependent conductance components

$$
g_{i j s}=R d J_{i j s} / d V \text {. }
$$

The $\mathrm{G}-\mathrm{V}$ characteristics for a symmetrical CDWM-I-CDWM tunnel junction are shown in Fig. 2, $a$ for the cases where the external magnetic field $H$ is absent or present. For the sake of definiteness, we shall restrict the numerical calculations in this Section to the case $\varphi=0$. The main properties of the overall conductance versus voltage dependence and its splitting in the magnetic field survive for arbitrary $\varphi$ (see a discussion in Appendix C). One can readily see that each square-root singularity from the positive or negative voltage branch is split into two peaks, whereas the step-like peculiarities remain unsplit. Moreover, the conductance in each split peak has a predominant (not unique) polarization indicated
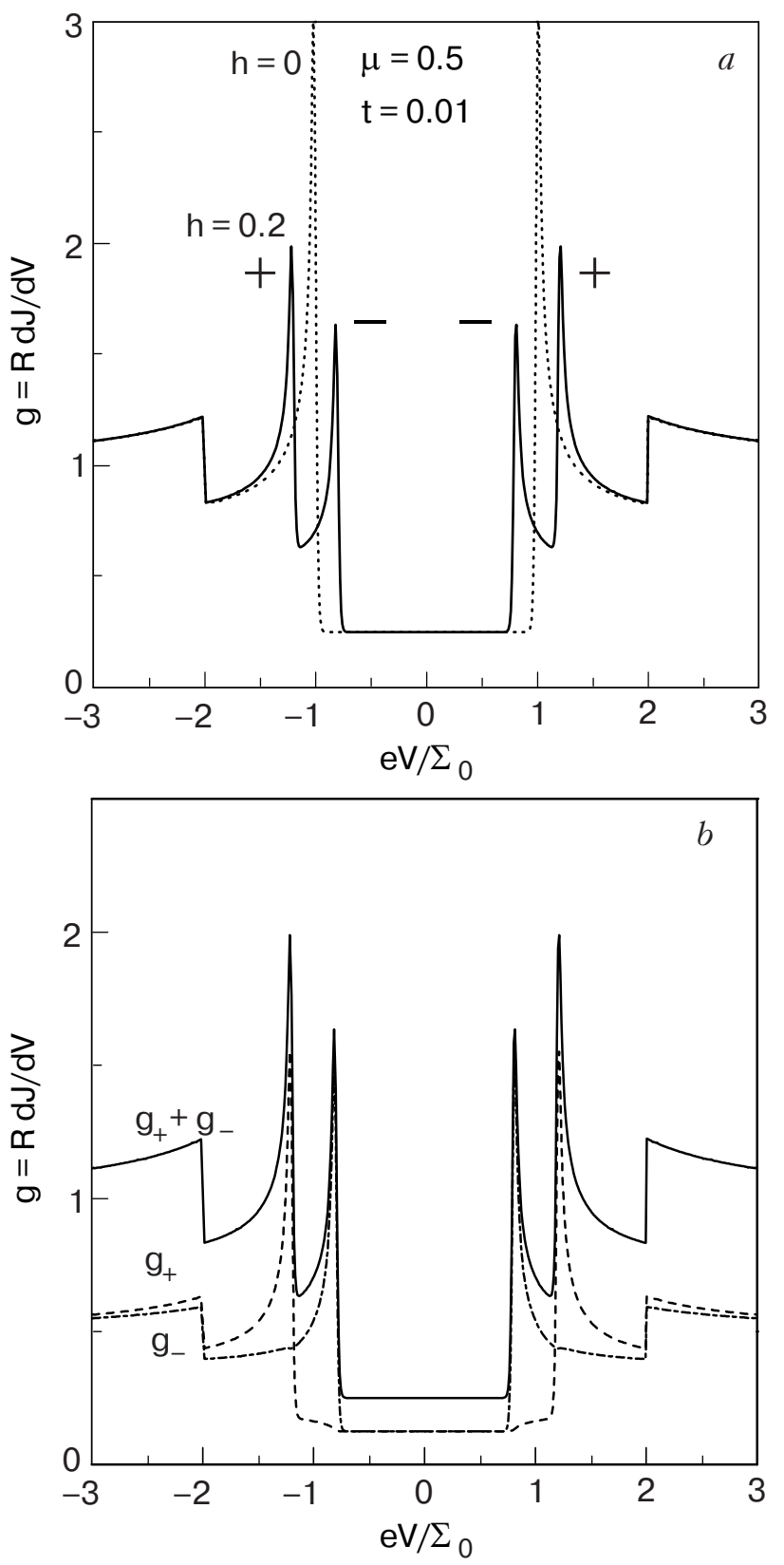

Fig. 2. The dimensionless conductance $g=R d J / d V$ of the symmetrical tunnel junction between similar CDW metals is shown as a function of the dimensionless bias voltage $e V / \Sigma_{0}$. Here $R$ is a tunnel junction resistance in the undistorted state above the critical temperature $T_{d}$ of the CDW transition, $\Sigma_{0}=\pi T_{d} / \gamma$ is the CDW gap at $T=0, J$ is the quasiparticle current, $e$ is the elementary charge, $\gamma=1.78 \ldots$ is the Euler constant, $T$ is temperature, $t=k_{B} T / \Sigma_{0}$ is the dimensionless temperature, $k_{B}$ is the Boltzmann constant, $h=\mu_{B}^{*} H / \Sigma_{0}, \mu=N_{d 0}(0) / N_{0}(0), N_{0}(0)=N_{n 0}(0)+N_{d 0}(0)$, $N_{d 0}(0)$ and $N_{n 0}(0)$ are the initial (above $T_{d}$ ) electronic densities of states on the $d$ and $n$ FS sections, respectively. The signs + and - indicate a predominant spin polarization of the peaks. (b) A decomposition of the total conductance $g$, displayed in panel $(a)$, into two summands $g_{s}$, each comprising contributions of current carriers with a corresponding spin polarization $s= \pm$. 
by a + or - sign. Figure $2, b$ illustrates a decomposition of the resulting $\mathrm{G}-\mathrm{V}$ characteristic for $H \neq 0$ into two components with different spin polarization of current carriers. One also sees a novel remarkable feature, namely, the peaks of the «t» component move apart in the magnetic field, whereas their «-» counterparts converge. Such a behavior differs drastically from that appropriate to S-I-N junctions, for which the $g_{+}(V)$ and $g_{-}(V)$ peaks move in opposite directions, irrespective of the voltage polarity. As to the tunneling through a $\mathrm{S}^{\prime}-\mathrm{I}-\mathrm{S}$ junction, the peak-to-peak separation does not depend on $H$ and is the same for either sign of $s[13,14]$. To explain the distinction between the superconducting and CDW cases, we should consider in detail each current constituent involved (recall that the $c d \pm$ and $d c \pm$ components were excluded from analysis for the symmetrical CDWM-I-CDWM configuration due to their mutual compensation).

First, as stems from the speculations in Appendix A, the tunneling between the gapped FS sections, as well as the transfer of the electron-hole pairs across the barrier, do not induce any peak splitting in the assumed absence of spin flips. This concerns the contributions $d d \pm, c c \pm, c d \pm$, and $d c \pm$ to both $J(V)$ and $G(V)$, and is similar to what happens in $\mathrm{S}^{\prime}-\mathrm{I}-\mathrm{S}$ junctions. At the same time, the «normal» $n n \pm$ components should reveal no noticeable magnetoresistance under the action of relatively small magnetic fields $H \leq 0.2 \Sigma_{0} \approx 2.8 \mu_{B}^{*} H_{p} / \sqrt{\mu}$, which are, e.g., far below the fields experimentally found necessary for orbital quantization in the organic material $\alpha$-(BEDT-TTF $)_{2}$ $\mathrm{KHg}(\mathrm{SCN})_{4}[27,51]$. Here, relationship (8) was taken into account. Had it not been for other components, the $\mathrm{G}-\mathrm{V}$ characteristics of CDWM-I-CDWM junctions would have possessed only feature points at $e V= \pm 2 \Sigma$, as it is the case for $\mathrm{S}-\mathrm{I}-\mathrm{S}$ tunneling $[4,8,9]$.

Now let us pass on to the tunnel processes that connect the $n$ FS section of one electrode and the $d$ FS section of the another one (Fig. 3). For the sake of definiteness we start our analysis with the «+»-polarization conductance. The components $d n+$ and $n d+$ are contributions to the $\mathrm{G}-\mathrm{V}$ characteristics of the type known from the theory of superconducting splitting $[13,14]$. Using this analogy, it can be shown that the dimensionless conductances $g_{d n^{+}, n d^{+}}=R d J_{d n^{+}, n d^{+}} / d V$ can be represented as

$$
\begin{gathered}
g_{d n+}(e V, H) \propto \int_{-\infty}^{\infty} d \omega K_{0}(\omega-e V) F^{\prime}(\omega, H), \\
g_{n d+}(e V, H) \propto \int_{-\infty}^{\infty} d \omega K_{0}^{\prime}(\omega) F(\omega-e V, H) .
\end{gathered}
$$

Here, $F^{(')}(\omega, H)=N_{d+}^{(')}(\omega, H)$, i.e., the densities of the gapped «t»-states for the relevant electrodes

$$
N_{d+}^{\left({ }^{\prime}\right)}(\omega, H)=N_{d 0}^{\left({ }^{\prime}\right)}(0) \frac{\left|\omega-\mu_{B}^{*} H\right| \theta\left(\left|\omega-\mu_{B}^{*} H\right|-\Sigma^{(')}\right)}{2 \sqrt{\left(\omega-\mu_{B}^{*} H\right)^{2}-\Sigma^{(') 2}}}
$$

[cf. Eq. (6)], and the kernels $K_{0}^{(')}$ are the derivatives of the kernel $K(\omega, e V)$ [Eq. (A.5)] in the integrand of Eq. (A.3). The quantities $K_{0}^{\left({ }^{\prime}\right)}$ are $\delta$-like functions with the maxima at the Fermi levels of the unprimed or primed electrodes, respectively, in the degenerate case considered. In the limiting case $T=0$, the $V$ dependence of the conductance $g_{d n+}(e V)$ coincides with that

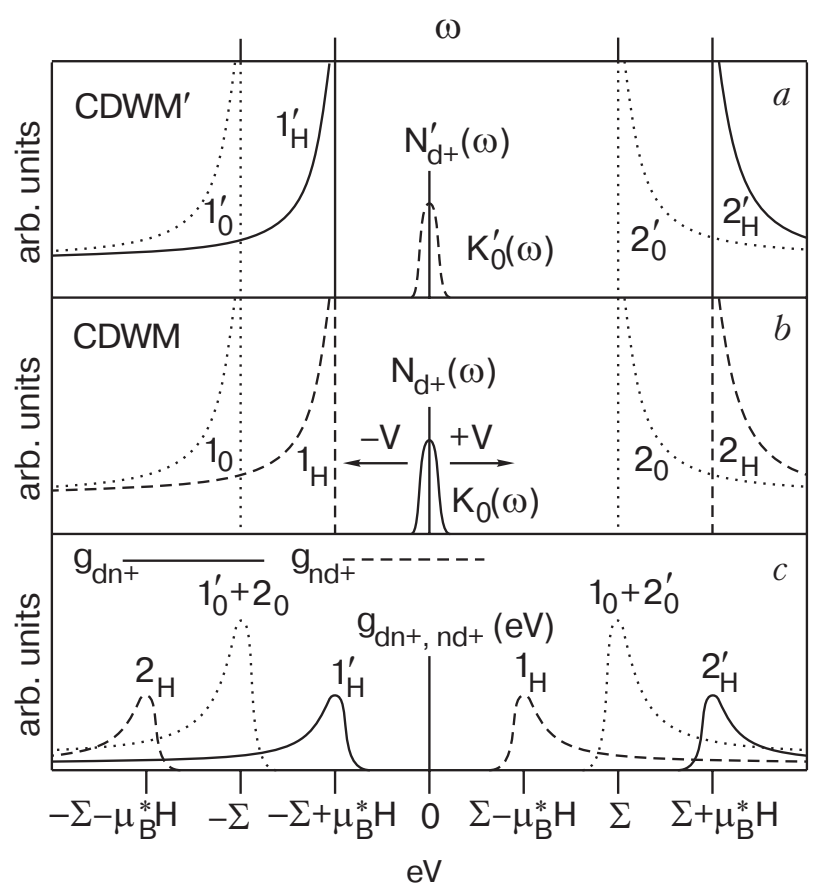

Fig. 3. The scheme of the peak spin-splitting for the conduction components $g_{d n+}$ and $g_{n d+}$ in the magnetic field $H$. (a) The energy dependence of the gapped DOSs $N_{d+}^{\prime}(\omega)$ for the primed electrode at $H=0$ (dotted curves, subscript 0 ) and $H \neq 0$ (solid curves, subscript $H$ ). The function $K_{0}^{\prime}(\omega)$ is a $T$-dependent kernel [Eq. (18)] originating from the Fermi distribution of the $n$ electrons. (b) The same as in panel $(a)$, with an accuracy up to notation, for the unprimed electrode. All elements of this panel are shifted with respect to those in panel $(a)$ by the value of $\mathrm{eV}$ if a bias voltage $V$ is applied. (c) The resulting contributions $g_{d n^{+}}$and $g_{n d^{+}}$to the $\mathrm{G}^{-} \mathrm{V}$ characteristic. Any peak in a certain conductance component appears if a DOS singularity in one panel is overlapped by the kernel function in the other one [those three relevant elements (the peak, the DOS, and the kernel) are drawn using the same style of the curve]. The numbering of the peaks corresponds to the «parent» DOS singularities in panels $(a)$ and $(b)$. See further explanations in the text. 
of $N_{d+}^{\prime}(e V, H)$ [see Eq. (17)], while $g_{n d+}(e V)$ becomes proportional to $N_{d_{+}}(-e V, H)$ [see Eq. (18)].

When $H=0$, the electrode Fermi levels are located at the center of the relevant CDW gaps and coincide in the absence of the bias voltage. The bias magnitudes, needed either to shift the Fermi level of the unprimed electrode (see Fig. 3,b, $K_{0}$ ) downwards in reference to the lower edge of the CDW gap of the other electrode (Fig. 3, $a, 1_{0}^{\prime}$, dotted) or to step it up with respect to the upper edge of the same gap (Fig. $3, a, 2_{0}^{\prime}$, dotted) are equal. Hence, the positions of relevant singularities in the term $g_{d n+}(e V)$ are equidistant from $V=0$ (Fig. 3,c, component $1_{0}^{\prime}$ of the combined peak $1_{0}^{\prime}+2_{0}$ and component $2_{0}^{\prime}$ of the combined peak $1_{0}+2_{0}^{\prime}$, dotted). The same is valid for the contribution $g_{n d+}(e V)$ [Eq. (18)] which contains peak $1_{0}$, positioned at the same bias as the $2_{0}^{\prime}$ one, and peak $2_{0}$, disposed at the same bias as the $1_{0}^{\prime}$ one. Their amplitudes are also pairwise equal, since for the symmetrical junction $K_{0}(\omega)=K_{0}^{\prime}(\omega)$ and $N_{d}(\omega)=N_{d}^{\prime}(\omega)$. Evidently, one cannot distinguish between contributions of the pair elements to the corresponding features of the $\mathrm{G}-\mathrm{V}$ characteristic, the latter therefore being unsplit (Fig. 3,c, dotted peaks $1_{0}^{\prime}+2_{0}$ and $1_{0}+2_{0}^{\prime}$ ).

When the magnetic field is switched on but the junction is not biased, the «+» subsystem in each electrode shifts upwards in energy by $+\mu_{B}^{*} H$ relative to their common Fermi level, which remains fixed (see Fig. 3, $a$, solid curves, and Fig. 3, $b$, dashed curves). Thus, different bias voltages with different $H$-driven offsets should be applied now to obtain peaks in either of conductance terms. In particular, the singularities in the $g_{d n+}(e V)$ component shift to $\pm \Sigma+\mu_{B}^{*} H$ positions (Fig. 3,c, solid peaks $1_{H}^{\prime}$ and $2_{H}^{\prime}$ ) and the singularities in the $g_{n d+}(\mathrm{eV})$ component shift to $\pm \Sigma-\mu_{B}^{*} H$ positions (Fig. $3, c$, dashed peaks $1_{H}$ and $2_{H}$ ). The nomenclature of peaks in Fig. 3,c coincides with that in Figs.3, $a$ or 3, $b$, explicitly indicating the gap edge responsible for each feature. Thus, either of the $\mathrm{G}-\mathrm{V}$ characteristic peaks, being combined in a zero magnetic field, splits into two smaller ones for $H \neq 0$. The peaks belonging to the $g_{d n+}$ or $g_{n d+}$ components shift symmetrically in opposite directions of the $V$ axis, which can be deduced directly from Eqs. (17) and (18). On the other hand, the apparent motion of the peak pairs $\left(1_{H}^{\prime}-1_{H}\right.$ and $\left.2_{H}^{\prime}-2_{H}\right)$, each originating from both $g_{d n+}$ and $g_{n d+}$ terms, is directed inwards and outwards, respectively. But even this rather complicated picture does not signify the end of the story.

The involvement of the $g_{c n+, n c+}$ components, directly descending from the electron-hole pairing, changes the situation radically and makes the resulting $\mathrm{G}-\mathrm{V}$ characteristics highly unconventional. In particular, the component $g_{c n+}$ is also of the form (17) but with another function $F^{\left({ }^{\prime}\right)}(\omega, H)=N_{d+}^{(')}(\omega, H)$. The term $g_{c n+}(V)$ has the same functional dependences and amplitude of singularity at the same voltage values as the component $g_{d n+}$ does, but, contrary to the latter and due to the non-trivial properties of the Green's function $G_{c}(\omega)$, it is antisymmetric with respect to $\omega$. As a consequence, the $g_{c n+}$ singularity enhances its counterpart of $g_{d n+}$ on the positive voltage branch and almost compensates the singularity of $g_{d n+}$ on the negative- $V$ branch, transforming it into a cusp. This is illustrated in Fig. 4. The resulting pattern moves as a whole along the $V$ axis towards larger positive $V$ if an external field $H$ is applied. At the same time, the $V$ dependence of the sum $g_{n d+}+g_{n c+}$ constitutes a specular reflection of the curve $g_{d n+}(V)+$ $+g_{c n+}(V)$ relative to the $g$ axis. Therefore, the overall conductance $g_{+}(V)$, which is a sum of all the four relevant contributions discussed above, has two peaks symmetrically moving apart and two cusps symmetrically crowding together along the $V$ axis as the magnetic field $H$ grows.

The conductance behavior obtained for the CDWM-I-CDWM sandwich is due to the fact that the FSs have both $n$ and $d$ sections. This means that the whole configuration can be viewed as a combination of two asymmetrical junctions. Hence, there are two unequal current components $J_{n d+}$ and $J_{d n+}$ con-

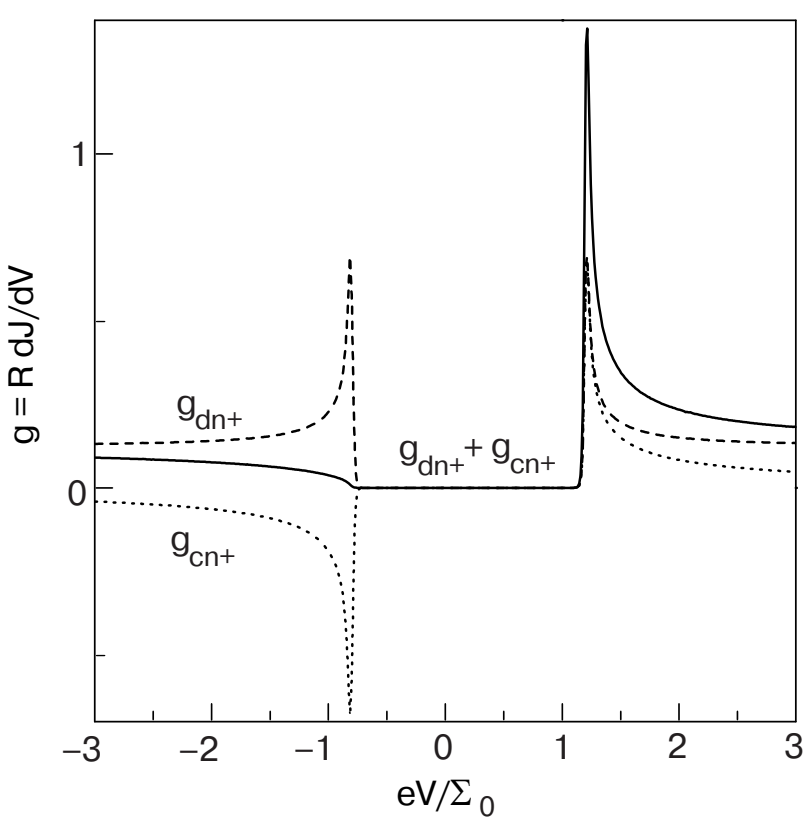

Fig. 4. An illustration of the component compensation: the selected contributions $g_{d n^{+}}, g_{c n^{+}}$to the overall conductance $g$ and their sum. An almost complete mutual compensation of the logarithmic singularities for negative $V$ and their amplification for positive $V$ is demonstrated. The relevant parameters are the same as in Fig. 2. 
necting the gapped and non-gapped quasiparticle subsystems. Moreover, the terms $J_{c n+}$ and $J_{n c+}$, related to the CDW pairing, result in the appearance of antisymmetrical conductance peaks. The interplay of all constituents leads to the effect described above.

An analysis of the sum $g_{d n-}+g_{c n-}+g_{n d-}+g_{n c-}$ is performed in the same way. The resulting two peaks converge symmetrically, whereas the two cusps symmetrically move apart, with increasing $H$. Since the cusps of one polarization superpose on the singularities of the other polarization and there is no spin filter in the circuit, the cusps may be inconspicuous against the background of the singularities (see Fig. 2,b). Taking into account the other components, not exhibiting a peak splitting of any nature, we obtain the $\mathrm{G}-\mathrm{V}$ characteristic shown in Fig. 2, which reveals four $H$-dependent polarized peaks at $\mathrm{eV}= \pm \Sigma \pm \mu_{B}^{*} H$ and two fixed jumps at $e V= \pm 2 \Sigma$.

The pattern obtained is a consequence of the choice $\varphi=0$ made above. If one assumes another realistic situation with the order parameter phase averaged out (see discussion in Sec. 3.2), components (14) and (15) should disappear, so that the $\mathrm{G}-\mathrm{V}$ characteristics will change substantially. To easily embrace all possible cases with varying $\varphi$, it is convenient to analyze all conductance components for $T=0$. The results are summarized in Appendix C.

\subsection{Influence of different factors on the $G-V$ characteristics}

Figure 2 distinctly reveals the main peculiarities of the $\mathrm{G}-\mathrm{V}$ characteristics for a symmetrical CDWM-I-CDWM junction. Namely, there exist discontinuities at $H$-independent locations $e V= \pm 2 \Sigma$, determined merely by a dielectric gap value. Besides, there are $H$-driven square-root singularities shifted by $\pm \mu_{B}^{*} H$ from the basic $e V= \pm \Sigma$ bias values. It is obvious that the larger is the magnetic field $H$, the stronger are the inward and outward displacements of the singular conductance peaks. As was clearly demonstrated above, the apparent splitting has a dual nature reflecting both the intrinsic configurational asymmetry of the junction concerned and the Zeeman effect.

Nonzero temperatures smear the overall curves $g(V)$ and especially the singular peaks. It is shown in Fig. 5, $a$ that these dependences are highly sensitive to the dimensionless parameter $t$. Therefore, to observe the predicted splitting in the magnetic field one should either heavily reduce $T$ or use CDWMs with large $T_{d}$ 's and hence CDW gaps $\Sigma_{0}$.

The influence of the control gapping parameter $\mu$ on the $\mathrm{G}-\mathrm{V}$ characteristics is demonstrated in Fig. 5,b. It is readily seen that the increase of $\mu$ reduces the minimal value of $g(V)$, determined by the $g_{n n \pm}$ contri-
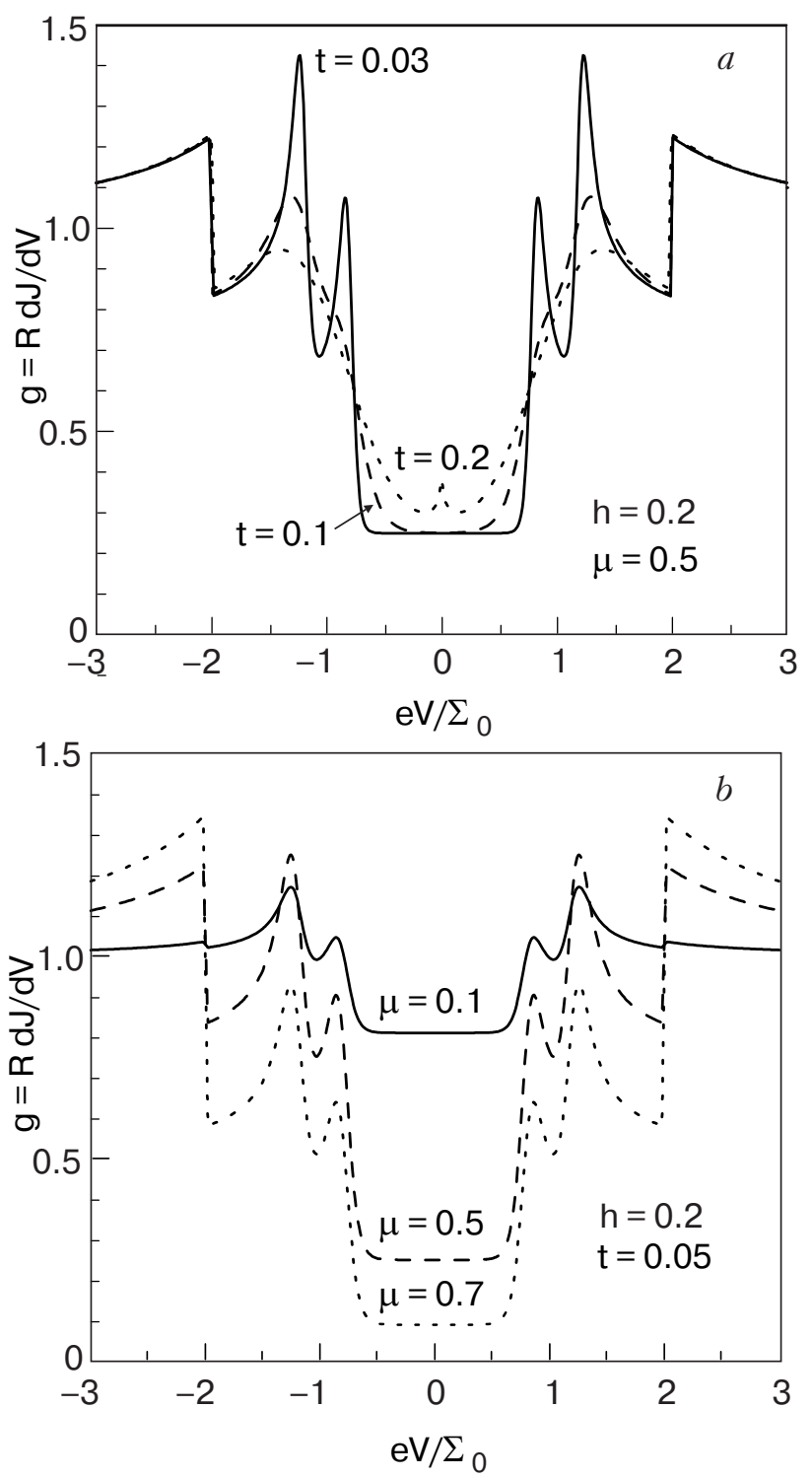

Fig. 5. The dependences $g\left(e V / \Sigma_{0}\right)$ for different $t(a)$ and different $\mu(b)$.

butions, and enhances the jump amplitude at $e V= \pm 2 \Sigma$. As stems from Fig. 5, $b$ and Eqs. (12)-(15), to improve the observability of the predicted splitting effect one should maximize the factor $\mu(1-\mu)$, i.e., those substances with $\mu$ close to 0.5 appear to be more promising.

\section{Discussion and conclusions}

The predicted splitting of the $\mathrm{G}-\mathrm{V}$ characteristic induced by the paramagnetic action of the magnetic field $H$ can be observed, in principle, for any CDW metal, i.e., the electron spectrum gapping should be incomplete, which is usually the case for a large number of low-dimensional Peierls metals with incommensurate CDWs. The specific requirement is to maintain a balance between $d$ and $n$ portions of the FS ( $\mu \approx 0.5$, see Sec. 3.2). This means that an external control of 
the parameter $\mu$ (e.g., by the applied pressure) may be crucial for the success of the experiment. The magnitude of the CDW gap $\Sigma$ defines the natural scale for the field $H$. Nevertheless, large $H$ and $\Sigma$ do not mean that the investigations can be carried out at high $T$. First, the spin splitting will be smeared and not resolved even for moderate $T / \Sigma$ (see Fig. 5). Second, in the close neighborhood of the paramagnetic limit it is possible to enter the region, where, in analogy with the case of superconductors, fluctuations [55] and the influence of the magnetic and spin-orbital scatterings [56], not covered by the present theory, may become important.

To make the predicted effects observable, it is crucial (at least in the symmetrical setup) for the components $J_{d n}$ and $J_{n d}$, describing the tunnel current linking $d$ and $n$ FS sections, to survive. In our phenomenological approach, when all matrix elements of the tunnel Hamiltonian [8] are considered equal (tunneling is not considered directional), it is the case. In the other extreme limit of the complete tunneling directionality [57], the components $J_{d n}$ and $J_{n d}$ may not exist and the spin splitting may disappear. In principle, any degree of directionality is possible. The actual realization of the intermediate situation stems from the analysis carried out for tunnel spectroscopic studies of high- $T_{c}$ oxides [58-63]. Therefore, one should consider the limit of no directionality and equal probability of all processes connecting different FS sections (a unique quantity $R$ ) as an idealized picture, so that for some junctions the feature points at $e V= \pm \Sigma$ might be weakened in comparison to those at $e V= \pm 2 \Sigma$. As a consequence, the spin splitting might be also partially suppressed.

The appearance of superconductivity for smaller $T<T_{c}<T_{d}$ in any specific CDW substance (see reviews $[11,12])$ may serve as a clear indication that this material is a metal rather than an insulator, and, therefore, of its ability to demonstrate the Zeeman spin splitting. Hence, the low-dimensional metals exhibiting $\mathrm{CDW}$ instabilities, such as $\mathrm{NbSe}_{3}, \mathrm{Nb}_{3} \mathrm{Te}_{4}$, $\mathrm{Li}_{0.9} \mathrm{Mo}_{6} \mathrm{O}_{17}, \mathrm{Tl}_{2} \mathrm{Mo}_{6} \mathrm{Se}_{6}$, layered dichalcogenides, alloys with the A15 and C15 structures, $\mathrm{Lu}_{5} \mathrm{Ir}_{4} \mathrm{Si}_{10}$, $\mathrm{P}_{4} \mathrm{~W}_{14} \mathrm{O}_{50}$, tungsten bronzes doped by alkali metals, and solid solutions $\mathrm{BaPb}_{1-x} \mathrm{Bi}_{x} \mathrm{O}_{3}$ may serve as good candidates. Other monophosphate bronzes $\left(\mathrm{PO}_{2}\right)_{4}\left(\mathrm{WO}_{3}\right)_{2 \mathrm{~m}}$, doped and undoped by alkalis, are also suitable partially gapped CDWMs $[64,65]$.

An important conjecture should be made concerning the magnitude of the CDW gaps. For superconductors, the ratio $2 \Delta(0) / k_{B} T_{C}$ is usually of the order or somewhat larger than the BCS value $2 \pi / \gamma \approx 3.52$ [4]. The only exception is $\mathrm{MgB}_{2}$. In that case, the very character of the superconductivity is as yet ambiguous and an intrinsic two-gap scenario is often accepted (see the relevant critical discussion of this concept in Refs. 66, 67). On the other hand, the observed dependence $\Sigma(T)$ in CDWMs and CDW insulators has a generalized BCS-like form. Namely, in the coordinates $\Sigma(T) / \Sigma(T=0)$ vs $T / T_{d}$, the data follow the Mühlschlegel curve, whereas the ratio $2 \Sigma(0) / k_{B} T_{d}$ essentially exceeds the BCS weak-coupling limit (such a behavior is described by the phenomenological scheme [68]). For example, this quantity is about 13 in the insulating $\mathrm{La}_{1.67} \mathrm{Sr}_{0.33} \mathrm{NiO}_{4}$ [69]. Layered dichalcogenides $2 H-\mathrm{TaSe}_{2}, 2 H-\mathrm{TaS}_{2}$ and $2 H-\mathrm{NbSe}_{2}$ are marked by extremely large values $2 \Sigma(0) / k_{B} T_{d}=15.2,15.4$, and 23.9, respectively [70]. In $\mathrm{NbSe}_{3}$, with its two CDW transitions at $T_{d}^{\text {low }}=59 \mathrm{~K}$ and $T_{d}^{\text {high }}=145 \mathrm{~K}[11,12]$, the respective ratios, as was shown by direct tunneling studies [71], fall into the ranges $2 \Sigma^{\text {low }}(0) / k_{B} T_{d}^{\text {low }} \approx$ $\approx 11.8-14.3$ and $2 \Sigma^{\text {high }}(0) / k_{B} T_{d}^{\text {high }} \approx 11.4-14.4$. Taking the observed Gaussian spread of the CDW gaps into account gives somewhat lower values $2 \Sigma^{\text {low }}(0) / k_{B} T_{d}^{\text {low }} \approx 9.2$ and $2 \Sigma^{\text {high }}(0) / k_{B} T_{d}^{\text {high }} \approx 8.2$ [72]. Larger gap-to- $T_{d}$ ratios are favorable for our purposes, since to clearly observe the splitting, one should avoid high temperatures during the experiment, although large gaps are convenient.

Superconducting cuprates can be suggested as another class of substances in which the CDW-triggered spin-splitting in the magnetic field can be observed. Two kinds of features testify that CDWs exist in a number of high- $T_{C}$ oxides. The first one is a dip-hump structure of $G(V)$ for voltages exceeding the positions of the superconducting gap maxima [72,73], while the other one is the so-called pseudogap, $\Sigma_{*}$, persisting both above and below $T_{c}[11,12,74-78]$. We think that the CDW origin of those peculiarities is quite plausible, whereas the most popular interpretation based on the precursor Cooper pairing (see, e.g., review [79] and references therein) should be rejected, at least because the applied magnetic field influences true gaps and the $\Sigma_{*}$ 's in a different way [80-82]. Moreover, the predominantly paramagnetic character of the magnetic field influence on the $\Sigma_{*}$ 's is attested by the existence of Zeeman scaling (proportionality) between the pseudogap-closing field $H_{\mathrm{pg}}$ and the characteristic pseudogap temperature $T^{*}$ resistively determined in $\mathrm{Bi}_{2} \mathrm{Sr}_{2} \mathrm{CaCu}_{2} \mathrm{O}_{8-y}$ [83,84]. It means that the huge orbital Meissner effect is absent for $\Sigma_{*}$, so that at least in $\mathrm{Bi}_{2} \mathrm{Sr}_{2} \mathrm{CaCu}_{2} \mathrm{O}_{8-y}$ it may be identified with $\Sigma$. We recommend recent comprehensive reviews $[11,12,74-78,85]$ to compare the arguments belonging to various analysts in this field.

Low $T$ of measurements may turn out to be a necessary condition for resolving the spin splitting of CDW gaps (pseudogaps). Since one can make more definite 
conclusions for pure CDW phase above $T_{c}$, it is advantageous to carry out investigations in magnetic field for substances with relatively low $T_{c}$ much smaller than both $T_{d}$ and $\Sigma(T)$. That is why we consider the experiments of Ref. 86 very important. Specifically, a well-resolved superconducting gap $\Delta$ and a pseudogap $\Sigma$ were found by the authors of Ref. 86 for $\mathrm{Bi}_{2}\left(\mathrm{Sr}_{2-x} \mathrm{La}_{x}\right) \mathrm{CuO}_{6+\delta}$ mesas with $10 \mathrm{~K} \leq T_{c} \leq 32 \mathrm{~K}$. In a search for the predicted effect in cuprates, one should look through substances in which the following conditions are satisfied: (i) a clear-cut resolution between $\Delta$ and $\Sigma[73,80-82,86-88]$, (ii) all the four coexistent features, positioned at $\pm \Sigma$ and $\pm 2 \Sigma$, should be manifested, and (iii) the temperature of observation must be as low as possible. The last requirement might not be so severe as it seems at the first glance, because the same $H$ that drives the Zeeman peak splitting would suppress superconductivity, making the CDW gap itself open for probing.

\section{Acknowledgements}

A.M.G. is grateful to the Japan Society for the Promotion of Science for support of his visit to the Hiroshima University (Grant ID No. S-03204) and to the Mianowski Foundation for support of his visit to Warsaw University. The research has been partly supported by the NATO grant PST.CLG.979446 and the grants COE (No. 13CE2002) and Scientific Research (No. 15540346) of the Ministry of Education, Culture, Sports, Science and Technology of Japan. The authors are also grateful to Jun Akimitsu (Tokyo), Serguei Brazovskii (Kyoto), Kenji Ishida (Kyoto), Yoshiteru Maeno (Kyoto), and Mai Suan Li (Warsaw) for fruitful discussions.

\section{Appendix A: Tunnel current components}

Let us consider the general case of two different CDWMs with relevant parameters $\left(\tilde{\Sigma}^{\prime}, \mu^{\prime}\right)$ and $(\tilde{\Sigma}, \mu)$ at both sides of the potential barrier created by an insulating interlayer. The current-voltage (I-V) characteristics $J(V)$ for the quasiparticle tunnel current in this junction are calculated by the tunnel Hamiltonian method $[4,10,40,42]$ in the first order of the perturbation scheme $[32,89]$. Under the assumption that there is no spin flipping while tunneling, the overall tunnel current $J$ can be described as consisting of the following 18 terms:

$$
\begin{aligned}
& 18 \text { terms: } J_{i j \pm} \propto \operatorname{Re} \int_{-\infty}^{\infty} d \omega^{\prime} \times \\
& \times \int_{-\infty}^{\infty} d \omega \frac{\operatorname{Im} G_{i s}^{\prime}\left(\omega^{\prime} \mp \mu_{B}^{*} H\right) G_{j s}\left(\omega \mp \mu_{B}^{*} H\right)}{\omega^{\prime}-\omega+e V+i 0},
\end{aligned}
$$

which correspond to various combinations of Green's functions and spin projections $(s= \pm)$ for the two electrodes. Here the quantities related to different electrodes are primed or unprimed, the subscripts $i$ and $j$ of the Green's functions $G(\omega)$ 's equal to $d$, $n$, or $c$ [see Eqs. (3)-(5) for notations], and the signs in the Green's functions' arguments are opposite to those of the spin projection. Hereafter, the potential of the primed electrode is taken as zero, so that the bias voltage $V$ comprises the electrostatic potential of the unprimed electrode. After standard calculations following the pattern of Refs. 5, 11, 12, we obtain the expression

$$
J=\sum_{\substack{i, j=d, n, c \\ s=+,-}} J_{i j s},
$$

with each component and the overall current depending on the bias voltage $V$, temperature $T$, and $H$. All current components $J_{i j s}$ have the general form

$$
\begin{aligned}
J_{i j \pm}= & \frac{1}{4 e R} \int_{-\infty}^{\infty} d \omega K Z_{i}\left(\omega \mp \mu_{B}^{*} H, \tilde{\Sigma}^{\prime}\right) \times \\
& \times Z_{j}\left(\omega \mp \mu_{B}^{*} H-e V, \tilde{\Sigma}\right) .
\end{aligned}
$$

The quantity

$$
R^{-1}=4 \pi e^{2} N_{0}^{\prime}(0) N_{0}(0)\left\langle\left|T_{\mathbf{p q}}\right|^{2}\right\rangle_{F S}
$$

is the conductance (inverse resistance) of the junction above $T_{d}$, where both electrodes are in the non-gapped state. The square of the modulus of the tunnel matrix element $T \mathbf{p q}$ is averaged over the FS, i.e., all matrix elements of the tunnel Hamiltonian are taken equal. Thus, we assume a unique tunnel resistance for every current component. From the physical point of view this means, in particular, no tunnel directionality, which is possible, in principle [58-63]. The factor

$$
K \equiv K(\omega, V, T)=\tanh \frac{\omega}{2 T}-\tanh \frac{\omega-e V}{2 T}
$$

stems from the Fermi distribution functions of the two electrodes.

Each $Z$ function in the integrand of Eq. (A.3) constitutes a product

$$
Z_{i}(\omega, \tilde{\Sigma})=z_{i}(\omega, \tilde{\Sigma}) f_{i}(\omega, \tilde{\Sigma})
$$

of one of the characteristic functions

$$
\begin{gathered}
z_{d}(\omega, \tilde{\Sigma})=\mu|\omega|, \\
z_{c}(\omega, \tilde{\Sigma})=\mu \operatorname{sgn}(\omega) \tilde{\Sigma}, \\
z_{n}(\omega, \tilde{\Sigma})=(1-\mu)|\omega|,
\end{gathered}
$$

and the factor 


$$
f_{i}(\omega, \tilde{\Sigma})=\frac{\theta(|\omega|-\Sigma)}{\sqrt{\omega^{2}-\Sigma^{2}}}
$$

which describes the presence ( $\tilde{\Sigma} \neq 0$ for $i=d$ and $c$ ) or absence $(\tilde{\Sigma}=0$ for $i=n)$ of gapping; $\theta(x)$ is the Heaviside step function. For $i=n$, the $Z_{n}$ function reduces to

$$
Z_{n}(\omega, \tilde{\Sigma})=1-\mu .
$$

When the gapping is absent in both electrodes $\left(\mu=\mu^{\prime}=0\right)$, the total current consists of $J_{n n \pm}$ components only and has a conventional Ohm's form $V / R$ in the whole voltage range, independent of $T$ and $H$. Of course, in the general case, Ohm's law is restored for large enough voltages substantially exceeding $\Sigma^{\prime}$ and $\Sigma$.

Tunnel currents between CDWMs across the barriers of different transparencies making allowance for the dependences of $J$ on $\varphi$ and $\varphi^{\prime}$ were studied in a number of papers [21,90-93]. Contrary to some of them [91-93], we shall consider the most general setup, when $\varphi$ and $\varphi^{\prime}$ are independent of each other. The situation when $\varphi=\varphi^{\prime}$ will stem from the general equations as a particular case.

Let us analyze the symmetry properties of different current components. It is convenient to start from the case $H=0$. Such an analysis has been done earlier [5], but to investigate below a more involved situation with nonzero $H$, it is necessary to carry out an additional examination. We shall use the notation $J_{i j 0}=$ $=J_{i j s}(H=0)$, since in this case $\omega_{ \pm}=\omega$ in the integrands of (A.3), and therefore $J_{i j+}(H=0)=$ $=J_{i j-}(H=0)$.

The components can be divided into symmetrical and asymmetrical ones with respect to the voltage $V$. The symmetrical components, for which an unusual relation $[5,54]$

$$
J_{i j 0}(-V)=J_{i j 0}(V)
$$

holds, are those that contain the «anomalous» Green's function $G_{c}$ once in the integrand [Eq. (A.1)]. These are $J_{d c 0}, J_{c d 0}, J_{n c 0}$, and $J_{c n 0}$. Other components, namely, $J_{d d 0}, J_{c c 0}, J_{n n 0}, J_{d n 0}$, and $J_{n d 0}$, satisfy the conventional equation [9]

$$
J_{i j 0}(-V)=-J_{i j 0}(V) .
$$

It is worthwhile mentioning that the integrand of $J_{c c 0}$ includes a product of two Green's functions $G_{c}$, the anomalous symmetry properties of which compensate each other.

The differential conductance $G(V) \equiv d J(V) / d V$ is a quantity of primary interest to experimentalists. The same is true for its symmetry properties. The dependence $G(V)$ and similar $V$ dependences of its compo- nents $G_{i j}$ will be henceforth called the conductance-voltage $(\mathrm{G}-\mathrm{V})$ characteristics. The symmetry relationships for $G_{i j 0}(V)$ are easily deduced from those for $J_{i j 0}(V)$. Namely, for the $d d 0, c c 0, n n 0, d n 0$, and $n d 0$ components, they have the standard form [9]

$$
G_{i j 0}(-V)=G_{i j 0}(V),
$$

whereas for the $d c 0, c d 0, n c 0$, and $c n 0$ terms, the symmetry properties are anomalous:

$$
G_{i j 0}(-V)=-G_{i j 0}(V) .
$$

Going to the case $H \neq 0$, we should consider the changes of the CDWM electron spectrum on both sides of the junction under the influence of the external magnetic field (see Fig. 1). As was indicated in Sec. 2.1.2, the Fermi levels (chemical potentials) in both electrodes, differing from each other by $e V$, remain practically intact when $H$ is switched on. Hence, the electron spectrum on the $n$ FS sections is also unchanged. On the other hand, the quasiparticle energy subbands with the spin projection $s=+$ on the $d$ FS sections in both electrodes shift upwards by the value $\mu_{B}^{*} H$, while the subbands with the projection $s=-$ shift downwards by the same value.

Since tunneling is assumed to preserve spin values, it is clear that a current component depends on $H$ only in the case where the subbands involved (one from the primed electrode and the other from the unprimed one) change their energy difference with increasing magnetic field. Relevant links are shown in Fig. 1 by dashed lines. Therefore, all 18 components can be divided into three groups. The first one contains those terms which do not depend on $H$. They are $J_{d d s}, J_{c c s}$, $J_{d c s}, J_{c d s}$, and $J_{n n s}$, and for them

$$
J_{i j s}(e V, H)=J_{i j s}(e V, 0) .
$$

The next group of current components, $J_{d n^{+}}, J_{c n^{+}}$, $J_{n d^{-}}$, and $J_{n c^{-}}$, is shifted towards larger voltages for $H \neq 0$, i.e.,

$$
J_{i j s}\left(e V-\mu_{B}^{*} H, H\right)=J_{i j s}(e V, 0) .
$$

The remaining terms $J_{d n^{-}}, J_{c n^{-}}, J_{n d^{+}}$, and $J_{n c^{+}}$move in the opposite direction of the $V$ axis, i.e.,

$$
J_{i j s}\left(e V+\mu_{B}^{*} H, H\right)=J_{i j s}(e V, 0) .
$$

Various links describing such $H$-dependent current components are specified by solid lines in Fig. 1.

Those components play a crucial role, because they lead to a new phenomenon revealed in junctions between CDWMs exposed to a magnetic field. Specifically, the overall tunnel current and peaks in $G(V)$, originating from the CDW gapping, are spin-split even in the symmetrical setup. This is unlike the total absence of splitting when both electrodes are 
superconducting, whatever the relative magnitudes of the superconducting gaps [13].

It is worthwhile to discuss one peculiarity, concerning the shifts (A.17) and (A.18) of the I-V (and, by implication, the $\mathrm{G}-\mathrm{V}$ ) characteristics along the $V$ axis. It happens that $J_{i j \pm}(e V=0, H \neq 0) \neq 0$ for those current components, although each component in the sum (A.2) becomes zero in the absence of $H$ and in the absence of voltage: $J_{i j 0}(e V=0)=0$. But this does not signify any violation of the laws of quasi-stationary electrodynamics, since only the total current (A.2), for which $J(e V=0, H \neq 0)=0$ irrespective of the magnitude of $H$, has physical meaning.

\section{Appendix B: Analytical expressions for tunnel current components in asymmetrical and symmetrical junctions at $\boldsymbol{T}=\mathbf{0}$ and $\boldsymbol{H}=\mathbf{0}$}

Here, the analytical expressions for tunnel current components across the insulating barrier between dissimilar CDWM electrodes at $T=0$ and in the absence of the magnetic field are calculated. The derivation of the corresponding expressions is straightforward although cumbersome. The final results are displayed below. Only the branches $V>0$ of the components of the $\mathrm{I}-\mathrm{V}$ characteristic are explicitly shown, because their negative $V$ counterparts can be easily obtained using the symmetry properties (A.12) and (A.13).

$$
\begin{gathered}
J_{d d}(V>0)=\frac{\mu^{\prime} \mu}{e R}\left\{\left[N+2 \sqrt{\Sigma^{\prime} \Sigma}\right] \mathbf{E}(\alpha)-\right. \\
\left.-\frac{4 \sqrt{\Sigma^{\prime} \Sigma}\left[N+\sqrt{\Sigma^{\prime} \Sigma}\right]}{\left[N+2 \sqrt{\Sigma^{\prime} \Sigma}\right]} \mathbf{K}(\alpha)\right\} \theta\left[e V-\left(\Sigma^{\prime}+\Sigma\right)\right] ; \\
=-\frac{4 \mu^{\prime} \mu \Sigma^{\prime} \Sigma \cos \varphi^{\prime} \cos \varphi \mathbf{K}(\alpha) \theta\left[e V-\left(\Sigma^{\prime}+\Sigma\right)\right]}{e R\left[N+2 \sqrt{\Sigma^{\prime} \Sigma}\right]} ;
\end{gathered}
$$

$J_{d n}(V>0)=\frac{1}{e R}\left\{\mu^{\prime}(1-\mu) \theta\left(e V-\Sigma^{\prime}\right) \sqrt{(e V)^{2}-\Sigma^{\prime 2}}\right\}$

$$
J_{n d}(V>0)=\frac{1}{e R}\left\{\mu\left(1-\mu^{\prime}\right) \theta(e V-\Sigma) \sqrt{(e V)^{2}-\Sigma^{2}}\right\} \text {. }
$$

$$
\begin{aligned}
J_{c d}(V>0)=\frac{2 \mu^{\prime} \mu \Sigma^{\prime} \Sigma \theta\left[e V-\left(\Sigma^{\prime}+\Sigma\right)\right]}{e R N} \cos \varphi^{\prime} \times \\
\times\left\{2 \Pi\left(\frac{\pi}{2}, \frac{e V-\Sigma^{\prime}-\Sigma}{e V-\Sigma^{\prime}+\Sigma}, k\right)-\mathbf{K}(k)\right\} ; \quad
\end{aligned}
$$

$$
\begin{gathered}
J_{d c}(V>0)=\frac{2 \mu^{\prime} \mu \Sigma^{\prime} \Sigma \theta\left[e V-\left(\Sigma^{\prime}+\Sigma\right)\right]}{e R N} \cos \varphi \times \\
\times\left\{-2 \Pi\left(\frac{\pi}{2}, \frac{e V-\Sigma^{\prime}-\Sigma}{e V+\Sigma^{\prime}-\Sigma}, k\right)+\mathbf{K}(k)\right\} ; \\
J_{c n}(V>0)=\frac{1}{e R} \mu^{\prime} \Sigma^{\prime} \cos \varphi^{\prime} \theta\left(e V-\Sigma^{\prime}\right) \times \\
\times \ln \left|\frac{e V+\sqrt{(e V)^{2}-\Sigma^{\prime 2}}}{\Sigma^{\prime}}\right| ; \\
J_{n c}(V>0)=\frac{1}{e R} \mu \Sigma \cos \varphi \theta(e V-\Sigma) \times \\
\times \ln \left|\frac{e V+\sqrt{(e V)^{2}-\Sigma^{2}}}{\Sigma}\right|
\end{gathered}
$$

Expression (11) for the current between $n$ FS sections conserves its form. Here $\mathbf{K}(x), \mathbf{E}(x)$ and $\mathbf{\Pi}(\pi / 2, x, y)$ are the complete elliptic integrals of the first, second, and third kind, respectively. Their arguments are

$$
k=\frac{\sqrt{(e V)^{2}-\left(\Sigma^{\prime}+\Sigma\right)^{2}}}{N}
$$

and

$$
\alpha=\frac{N-2 \sqrt{\Sigma^{\prime} \Sigma}}{N+2 \sqrt{\Sigma^{\prime} \Sigma}},
$$

whereas the quantity $N$ is equal to

$$
N=\sqrt{(e V)^{2}-\left(\Sigma^{\prime}-\Sigma\right)^{2}} .
$$

One can readily see that the analytical expressions for the tunnel current between dissimilar CDWMs differ substantially from their well-known counterparts in the case of the quasiparticle current between different superconductors [8]. Namely, there is a single term $J_{s c}(V)$ for the superconducting junction coinciding with our component $J_{d d}(V)$ with an accuracy up to substitution of the superconducting gaps $\Delta^{\prime}$ and $\Delta$ for the CDW ones $\Sigma^{\prime}$ and $\Sigma$, while setting $\mu^{\prime}=\mu=1$. Extra terms originate from the pairwise combinations of the Green's functions (3), (4) and (5) appropriate to the currently investigated case of the junction involving CDWMs.

The main qualitative distinction between superconductor- and CDWM-based junctions consists in the different form of the feature points. There is one break point in the superconducting junction at $e V=\Delta^{\prime}+\Delta$, where the current steeply changes from zero to the value

$$
\Delta J_{s c}=\frac{\pi \sqrt{\Delta^{\prime} \Delta}}{2 e R} .
$$


At the same time, from Eqs. (B.1), (B.2), (B.5) and (B.6) it comes about that

$$
\Delta J\left(e V=\Sigma^{\prime}+\Sigma\right)=\frac{\pi \mu^{\prime} \mu \sqrt{\Sigma^{\prime} \Sigma}}{2 e R}\left(1+\cos \varphi^{\prime}\right)(1-\cos \varphi) .
$$

One should note that there is an intrinsic asymmetry in Eq. (B.13), i.e., $\Delta J$ depends on each of the phases $\varphi^{\prime}$ and $\varphi$ separately and in a different way. Both phase factors $\cos \varphi^{\prime}$ and $\cos \varphi$ will change their signs for the opposite voltage polarity $V<0$. A similar asymmetry takes place in an asymmetrical junction between CDW conductors [5]. The jump disappears in the particular case of an excitonic insulator, where each of the phase angles is either 0 or $\pi$, contrary to what is appropriate to the superconducting tunneling.

The emergence of root singularities in $J_{d n}, J_{n d}, J_{c n}$ and $J_{n c}$ components [see Eqs. (B.3), (B.5), (B.7) and (B.8), respectively] is another important phenomenon appropriate to asymmetrical junctions involving CDWMs.

The phase dependences of the current components $J_{c d}, J_{d c}, J_{c n}$ and $J_{n c}$, represented by Eqs. (B.5), (B.6), (B.7) and (B.8), respectively, were obtained assuming definite constant values of the phases $\varphi^{\prime}$ and $\varphi$ for both electrodes. Actually, an averaging of the currents over the junction cross section, as a consequence of the phase randomness, may wipe out these terms. To preserve them, the usage of the break-junction technique confining the contact area would be of benefit. Nevertheless, the resulting current $J(V)$ would differ substantially from its superconducting analog even in this case. In particular, the jump $\Delta J\left(e V=\Sigma^{\prime}+\Sigma\right)$, expressed by Eq. (B.13), disappears, contrary to what stems from the BCS theory and experiments carried out for $\mathrm{S}^{\prime}-\mathrm{I}-\mathrm{S}$ junctions [9]. Another very important phenomenon that survives the averaging is the square-root dependence of the components $J_{d n}$ and $J_{n d}$ on the voltage [see Eqs. (B.3) and (B.4)].

The general results (B.1)-(B.8) can be substantially simplified in the case of identical CDW parameters in both electrodes $\left(\tilde{\Sigma}^{\prime}=\tilde{\Sigma}\right.$ and $\left.\mu^{\prime}=\mu\right)$. In that case the expression for the tunnel current takes the following form:

$$
\begin{gathered}
J(V>0)=\frac{1}{e R}\left\{(1-\mu)^{2} e V+2 \mu(1-\mu) \theta(e V-\Sigma) \times\right. \\
\times \sqrt{(e V)^{2}-\Sigma^{2}}+\mu^{2} \theta(e V-2 \Sigma) \times \\
\left.\times\left[(e V+2 \Sigma) \mathbf{E}\left(\alpha_{e}\right)-\frac{4 \mathbf{K}\left(\alpha_{e}\right) \Sigma\left[e V+\Sigma\left(1+\cos ^{2} \varphi\right)\right]}{e V+2 \Sigma}\right]\right\} .
\end{gathered}
$$

Here

$$
\alpha_{e}=\frac{e V-2 \Sigma}{e V+2 \Sigma} .
$$

For the pinned phase of the commensurate excitonic insulator $(\varphi=0$ or $\pi)$, the results of our previous work [5] are reproduced.

One can imagine a plausible situation wherein a tunnel current is assembled from a large enough contact area, so that the CDW phases vary substantially over the contact plane. Then all $\varphi$-dependent terms should be averaged out. This means that all components directly involving CDW pairing amplitude, i.e., possessing at least one $c$-subscript, must vanish in an asymmetrical configuration. For the CDWM-I-CDWM junction, this will result in a substitution of $1 / 2$ for $\cos ^{2} \varphi$.

\section{Appendix C: Tunnel conductance in a magnetic field at $\boldsymbol{T}=\mathbf{0}$}

It stems from the basic equations (9)-(15) that the $\mathrm{C}-\mathrm{V}$ characteristics for $\mathrm{CDWM}^{\prime}-\mathrm{I}-\mathrm{CDWM}$ junctions should differ substantially from their counterparts for $\mathrm{S}^{\prime}-\mathrm{I}-\mathrm{S}$ tunnel structures. Moreover, CDWM'-I-CDWM junctions exhibit peculiar Zeeman splitting (see Sec. 3.1) totally absent for currents between superconducting electrodes. Below, analytical formulas for $G_{ \pm}(V)$ components are represented as direct illustrative evidence for the predicted phenomena. Likewise in Appendix B only the branches $V>0$ of the components of the $\mathrm{C}-\mathrm{V}$ characteristics are explicitly shown.

In particular, the field-independent terms of the total conductance have the form

$$
\begin{aligned}
& G_{d d+}(V>0)+G_{c c+}(V>0)=G_{d d-}(V>0)+G_{c c-}(V>0)=\frac{\mu^{2}}{2 R} \theta(e V-2 \Sigma) \times \\
& \times\left[\mathbf{E}\left(\alpha_{e}\right) \frac{(e V)^{2}-2 \Sigma^{2}\left(1+\cos ^{2} \varphi\right)}{e V(e V-2 \Sigma)}+4 \Sigma \mathbf{K}\left(\alpha_{e}\right) \frac{\Sigma\left(1+\cos ^{2} \varphi\right)-e V}{(e V)^{2}-4 \Sigma^{2}}\right],
\end{aligned}
$$




$$
G_{n n+}(V>0)=G_{n n-}(V>0)=\frac{(1-\mu)^{2}}{2 R} .
$$

The notations here coincide with those of Appendix B. Other components split in a magnetic field and appear as

$$
\begin{aligned}
& G_{d n \pm}(V>0)= \\
& =\frac{\mu(1-\mu)\left(e V \pm \mu_{B}^{*} H\right) \theta\left(e V \pm \mu_{B}^{*} H-\Sigma\right)}{2 R \sqrt{\left(e V \pm \mu_{B}^{*} H\right)^{2}-\Sigma^{2}}}, \\
& G_{n d \pm}(V>0)= \\
& =\frac{\mu(1-\mu)\left(e V \mp \mu_{B}^{*} H\right) \theta\left(e V \mp \mu_{B}^{*} H-\Sigma\right)}{2 R \sqrt{\left(e V \mp \mu_{B}^{*} H\right)^{2}-\Sigma^{2}}},(\mathrm{C} .4) \\
& G_{c n \pm}(V>0)=-\frac{\mu(1-\mu) \Sigma \cos \varphi \theta\left(e V \mp \mu_{B}^{*} H-\Sigma\right)}{2 R \sqrt{\left(e V \mp \mu_{B}^{*} H\right)^{2}-\Sigma^{2}}}, \\
& G_{n c \pm}(V>0)=\frac{\mu(1-\mu) \Sigma \cos \varphi \theta\left(e V \pm \mu_{B}^{*} H-\Sigma\right)}{2 R \sqrt{\left(e V \pm \mu_{B}^{*} H\right)^{2}-\Sigma^{2}}} .
\end{aligned}
$$

A sum of components (C.1)-(C.6) gives the overall conductance $G(V)$, its shape shown in Fig. 2.

It comes about from Eqs. (C.1) and (A.14) that

$$
\Delta G(e V= \pm 2 \Sigma)=\frac{\pi \mu^{2}}{2 R},
$$

regardless of the order parameter phase $\varphi$. It is necessary to underline that the tunneling conductance for the superconducting symmetrical junction is determined by quite a different expression $[8,10]$,

$$
\begin{aligned}
G_{s c}(V>0)= & \frac{\theta(e V-2 \Delta)}{(e V-2 \Delta) R}\left[\mathbf{E}\left(\alpha_{s}\right) \frac{(e V)^{2}-2 \Delta^{2}}{e V}+\right. \\
& \left.+\mathbf{K}\left(\alpha_{s}\right) \frac{4 \Delta(e V-\Delta)}{e V+2 \Delta}\right],
\end{aligned}
$$

where $\Delta$ is the superconducting gap and

$$
\alpha_{s}=\frac{e V-2 \Delta}{e V+2 \Delta} .
$$

The conductance $G_{s c}(V)$ diverges when $\mathrm{eV}$ tends to $2 \Delta$ from above:

$$
G_{s c}(V) \underset{e V \rightarrow 2 \Delta+0}{\longrightarrow} \frac{\pi \Delta}{(e V-2 \Delta) R} .
$$

The distinction between properties (C.7) and (C.10) is due to the fact that the pertinent tunnel current in superconductors comprises a direct one-term convolu- tion of the Fermi-distribution factor and two gapped DOSs. On the other hand, for CDWMs, the singularities in the terms $G_{d d s}(V)$ at $e V= \pm 2 \Sigma$ are to a certain extent compensated by the contributions from the terms $G_{c c s}(V)$ formed by two «intersection» Green's functions $G_{c}[5,11,12]$.

As for the spin-split peaks at two times smaller voltages $e V= \pm \Sigma$ in $G(V)$ for CDWM-I-CDWM tunnel junctions, they should be observed only for partially gapped metals. Therefore, such substances, as, e.g., TTF-TCNQ, $\left(\mathrm{TaSe}_{4}\right)_{2} \mathrm{I}, \quad \sigma-\mathrm{TaS}_{3}$, or $\mathrm{K}_{0.33} \mathrm{MoO}_{3}$, which are insulating below $T_{d}$ [3], can not reveal the predicted phenomenon of anomalous magnetic-field-induced splitting.

1. B.I. Halperin and T.M. Rice, Solid State Phys. 21, 115 (1968).

2. Yu.V. Kopaev, Trudy Fiz. Inst. Akad. Nauk SSSR 86, 3 (1975).

3. G. Grüner, Density Waves in Solids, Addison-Wesley Publishing Company, Reading, Massachusetts (1994).

4. A.A. Abrikosov, Fundamentals of the Theory of Metals, North-Holland, Amsterdam (1987).

5. A.M. Gabovich and A.I. Voitenko, Phys. Rev. B52, 7437 (1995).

6. A.I. Anselm, Introduction into the Semiconductor Theory, Nauka, Moscow (1978) (in Russian).

7. A.M. Gabovich, A.I. Voitenko, M.S. Li, H. Szymczak, and M. Pękała, in: Physics of Spin in Solids: Materials, Methods and Applications, S. Halilov (ed.), Kluwer, Dordrecht (2004), p. 25.

8. D.H. Douglass, Jr. and L.M. Falicov, Progr. Low Temp. Phys. 4, 97 (1964).

9. E.L. Wolf, Principles of Electron Tunneling Spectroscopy, Oxford University Press, New York (1985).

10. G.D. Mahan, Many-Particle Physics, Kluwer Academic, New York (2000).

11. A.M. Gabovich and A.I. Voitenko, Fiz. Nizk. Temp. 26, 419 (2000) [Low Temp. Phys. 26, 305 (2000)].

12. A.M. Gabovich, A.I. Voitenko, and M. Ausloos, Phys. Rep. 367, 583 (2002).

13. R. Meservey and P.M. Tedrow, Phys. Rep. 238, 173 (1994).

14. P. Fulde, Adv. Phys. 22, 667 (1973).

15. R.H. McKenzie, cond-mat/9706235.

16. A. Bjeliš, D. Zanchi, and G. Montambaux, condmat/9909303.

17. N. Harrison, Phys. Rev. Lett. 83, 1395 (1999).

18. J.S. Qualls, L. Balicas, J.S. Brooks, N. Harrison, L.K. Montgomery, and M. Tokumoto, Phys. Rev. B62, 10008 (2000).

19. S.J. Williamson, C.S. Ting, and H.K. Fung, Phys. Rev. Lett. 32, 9 (1974).

20. M. Matos, G. Bonfait, R.T. Henriques, and M. Almeida, Phys. Rev. B54, 15307 (1996).

21. S.N. Artemenko and A.F. Volkov, Zh. Éksp. Teor. Fiz. 87, 691 (1984) [Sov. Phys. JETP 60, 395 (1984)]. 
22. I.V. Krive, A.S. Rozhavskii, and I.O. Kulik, Fiz. Nizk. Temp. 12, 1123 (1986) [Sov. J. Low Temp. Phys. 12, 635 (1986)].

23. S.N. Artemenko and A.F. Volkov, in: Charge Density Waves in Solids, L.P. Gor'kov and G. Grüner (eds.), North-Holland, Amsterdam (1985), p. 365.

24. N. Harrison, Phys. Rev. B66, 121101 (2002).

25. D. Zanchi, A. Bjeliš, and G. Montambaux, Phys. Rev. B53, 1240 (1996).

26. A.G. Lebed, Phys. Rev. Lett. 88, 177001 (2002).

27. D. Andres, M.V. Kartsovnik, P.D. Grigoriev, W. Biberacher, and H. Müller, Phys. Rev. B68, 201101 (2003).

28. T. Ishiguro and K. Yamaji, Organic Superconductors, Springer Verlag, Berlin (1990).

29. G. Bilbro and W.L. McMillan, Phys. Rev. B14, 1887 (1976).

30. R.R. Guseinov and L.V. Keldysh, Zh. Eksp. Teor. Fiz. 63, 2255 (1972).

31. A.M. Gabovich, E.A. Pashitskii, and A.S. Shpigel, Fiz. Tverd. Tela 18, 3279 (1976) [Sov. Phys. Solid State 18, 1911 (1976)].

32. A.M. Gabovich and A.I. Voitenko, J. Phys.: Condens. Matter 9, 3901 (1997).

33. A.M. Gabovich and A.I. Voitenko, Phys. Rev. B56, 7785 (1997)

34. High Temperature Superconductivity, V.L. Ginzburg and D.A. Kirzhnitz (eds.), Consultants Bureau, New York (1982).

35. J. Frenkel, Phys. Rev. 36, 1604 (1930).

36. Ya.I. Frenkel, Wave Mechanics, Volume 1, State Technical-Theoretical Publishing House, LeningradMoscow (1934) (in Russian).

37. A. Sommerfeld and H. Bethe, Elektronentheorie der Metalle, Springer Verlag, Berlin (1933).

38. A.I. Khachaturov, E. Hatta, and V.M. Svistunov, J. Phys. Soc. Jpn. 72, 131 (2003).

39. J. Bardeen, Phys. Rev. Lett. 6, 57 (1961).

40. M.H. Cohen, L.M. Falicov, and J.C. Phillips, Phys. Rev. Lett. 8, 316 (1962).

41. L. Solymar, Superconductive Tunneling and Applications, Chapman and Hall, London (1972).

42. I.O. Kulik and I.K. Yanson, Josephson Effect in Superconducting Tunnel Structures, Nauka, Moscow (1970) (in Russian).

43. L.D. Landau and E.M. Lifshits, Statistical Physics. Part 1, Nauka, Moscow (1976) (in Russian).

44. S.V. Vonsovskii, Magnetism. Magnetic Properties of Dia-, Para-, Ferro-, Antiferro- and Ferrimagnetics, Nauka, Moscow (1971) (in Russian).

45. A.M. Gabovich, Fiz. Tverd. Tela 25, 3179 (1983).

46. A.M. Gabovich and A.I. Voitenko, Phys. Status Solidi B133, 135 (1986).

47. A.M. Clogston, Phys. Rev. Lett. 9, 266 (1962).

48. B.S. Chandrasekhar, Appl. Phys. Lett. 1, 7 (1962).

49. A.M. Gabovich, A.S. Gerber, and A.S. Shpigel, Phys. Status Solidi B141, 575 (1987).

50. J. Singleton, Rep. Prog. Phys. 63, 1111 (2000).
51. D. Andres, M.V. Kartsovnik, W. Biberacher, H. Weiss, E. Balthes, H. Müller, and N. Kushch, Phys. Rev. B64, 161104 (2001).

52. A.G. Lebed, Pis'ma Zh. Éksp. Teor. Fiz. 78, 170 (2003).

53. J.R. Schrieffer, in: Tunneling Phenomena in Solids, E. Burstein and S. Lundqvist (eds.), Plenum Press, New York (1969), p. 287.

54. A.M. Gabovich, Fiz. Nizk. Temp. 19, 1098 (1993) [Low Temp. Phys. 19, 779 (1993)].

55. P.M. Tedrow and R. Meservey, Phys. Rev. B16, 4825 (1977).

56. R.C. Bruno and B.B. Schwartz, Phys. Rev. B8, 3161 (1973).

57. W.A. Harrison, Phys. Rev. 123, 85 (1961).

58. M. Ledvij and R.A. Klemm, Phys. Rev. B51, 3269 (1995).

59. Z. Yusof, J.F. Zasadzinski, and L. Coffey, Phys. Rev. B58, 514 (1998).

60. Y.-M. Nie and L. Coffey, Phys. Rev. B59, 11982 (1999).

61. G.B. Arnold and R.A. Klemm, Phys. Rev. B62, 661 (2000).

62. R.A. Klemm, Phys. Rev. B67, 174509 (2003).

63. J.E. Dowman, M.L. A. MacVicar, and J.R. Waldram, Phys. Rev. 186, 452 (1969).

64. M. Greenblatt, in: Physics and Chemistry of Low-Dimensional Inorganic Conductors, C. Schlenker, J. Dumas, M. Greenblatt, and S. van Smaalen (eds.), Plenum Press, New York (1996), p. 15.

65. V. Bondarenko, J.W. Brill, J. Duma, and C. Schlenker, Solid State Commun. 129, 211 (2004).

66. A.M. Gabovich, M.S. Li, M. Pękała, H. Szymczak, and A.I. Voitenko, J. Phys.: Condens. Matter 14, 9621 (2002).

67. T. Ekino, T. Takasaki, T. Muranaka, J. Akimitsu, and H. Fujii, Phys. Rev. B67, 094504 (2003).

68. H. Padamsee, J. E. Neighbor, and C. A. Shiffman, J. Low Temp. Phys. 12, 387 (1973).

69. T. Katsufuji, T. Tanabe, T. Ishikawa, Y. Fukuda, T. Arima, and Y. Tokura, Phys. Rev. B54, 14230 (1996).

70. C. Wang, B. Giambattista, C.G. Slough, R.V. Coleman, and M.A. Subramanian, Phys. Rev. B42, 8890 (1990).

71. T. Ekino and J. Akimitsu, Jpn. J. Appl. Phys. Lett. 26, Supplement 26-3, 625 (1987).

72. T. Ekino and J. Akimitsu, Physica B194-196, 1221 (1994).

73. T. Ekino, S. Hashimoto, T. Takasaki, and H. Fujii, Phys. Rev. B64, 092510 (2001).

74. T. Timusk and B. Statt, Rep. Prog. Phys. 62, 61 (1999).

75. J.C. Campuzano, M.R. Norman, and M. Randeria, cond-mat/0209476.

76. T. Timusk, Solid State Commun. 127, 337 (2003).

77. J.C. Phillips, A. Saxena, and A.R. Bishop, Rep. Prog. Phys. 66, 2111 (2003).

78. A. Damascelli, Z. Hussain, and Z-X. Shen, Rev. Mod. Phys. 75, 473 (2003). 
79. V.M. Loktev, R.M. Quick, and S.G. Sharapov, Phys. Rep. 349, 1 (2001).

80. A. Yurgens, D. Winkler, T. Claeson, S.-J. Hwang, and J.-H. Choy, Int. J. Mod. Phys. B13, 3758 (1999).

81. M. Suzuki and T. Watanabe, Phys. Rev. Lett. 85, 4787 (2000).

82. V.M. Krasnov, A. E. Kovalev, A. Yurgens, and D. Winkler, Phys. Rev. Lett. 86, 2657 (2001).

83. T. Shibauchi, L. Krusin-Elbaum, M. Li, M.P. Maley, and P. H. Kes, Phys. Rev. Lett. 86, 5763 (2001).

84. L. Krusin-Elbaum, T. Shibauchi, and C.H. Mielke, Phys. Rev. Lett. 92, 097005 (2004).

85. Y. Yanase, T. Jujo, T. Nomura, H. Ikeda, T. Hotta, and K. Yamada, Phys. Rep. 387, 1 (2003).

86. A. Yurgens, D. Winkler, T. Claeson, S. Ono, and Y. Ando, Phys. Rev. Lett. 90, 147005 (2003).
87. T. Ekino, Y. Sezaki, and H. Fujii, Phys. Rev. B60, 6916 (1999)

88. V.M. Krasnov, A. Yurgens, D. Winkler, P. Delsing, and T. Claeson, Phys. Rev. Lett. 84, 5860 (2000).

89. A.I. Larkin and Yu.N. Ovchinnikov, Zh. Ekksp. Teor. Fiz. 51, 1535 (1966) [Sov. Phys. JETP 24, 1035 (1966)].

90. K.M. Munz and W. Wonneberger, Z. Phys. B79, 15 (1990).

91. M.I. Visscher and G.E.W. Bauer, Phys. Rev. B54, 2798 (1996).

92. K. Sano, Progr. Theor. Phys. 109, 11 (2003).

93. K. Sano, Physica E18, 245 (2003). 